\title{
A INDUÇÃo AFETIVA EM CENÁRIOS DE REALIDADE VIRTUAL: AVALIAÇÃO DA SENSAÇÃO DE PRESENÇA
}

INDUCTION AFFECTIVE SCENARIOS VIRTUAL REALITY: ASSESSMENT OF THE SENSATION OF PRESENCE

INDUCCIÓN AFECTIVA EN ESCENARIOS DE REALIDAD VIRTUAL: EVALUACIÓN DE LA SENSACIÓN DE PRESENCIA

Ana Ribeiro* Luis Monteiro**

\section{RESUMO}

A Realidade Virtual (RV) tem sido utilizada na indução de emoções associadas a respostas emocionais de maior validade ecológica. A presença, enquanto experiência de "estar lá", num determinado ambiente mediado, tornou-se intimamente associada à RV. O presente trabalho tem como objetivo verificar se a visualização dos Cenários de Indução Afetiva $3 \mathrm{D}$ induz uma maior sensação de presença comparativamente aos seus homólogos apresentados com a tecnologia 2D. Apresentamos a 30 participantes saudáveis os Cenários de Indução Afetiva $3 \mathrm{D}$ em ambas as modalidades de visualizaçáo ( $2 \mathrm{D}$ vs. $3 \mathrm{D}$ ), simultaneamente efetuavam-se registros psicofisiológicos da Atividade Eletrodérmica e do Ritmo Cardíaco e, no final da visualização de cada cenário, aplicamos a escala de dominância do Self-Assessment Manikin adaptada para medir a presença, bem como o Slater-Usoh-Steed Questionnaire, que é um instrumento de autorrelato para avaliação da sensação de presença provocada. Os resultados globais sugerem que Cenários de Indução Afetiva 3D induzem uma maior sensação de presença, comparativamente aos seus homólogos apresentados na tecnologia 2D. Verificamos ainda que o cenário de valência desagradável provoca maior sensaçáo de presença comparativamente com os cenários de valência agradável e neutro.

Palavras-chave: presença; realidade virtual; cenários de indução afetiva 3D; condição emocional.

\footnotetext{
* Cespu, Instituto Superior de Ciências da Saúde - Norte, Gandra, Porto, Portugal.

** Cespu, Instituto Superior de Ciências da Saúde - Norte, Gandra, Porto, Portugal.
} 


\section{Abstract}

Virtual reality (VR) has been used to induce emotions, emotional responses associated with greater ecological validity. The presence, while the experience of "being there" in a given environment mediated, became closely associated with the VR. This study aims to verify, that viewing 3D Affective Induction Scenarios induce a greater sense of presence compared to their counterparts presented with 2D technology. 30 healthy participants observed the 3D Affective Induction Scenarios on both types of display (2D vs. 3D), while simultaneous records were made of Electrodermal Activity and the Heart Rate and at the end of each scenario, we used the dominance scale Self-Assessment Manikin adapted to the presence and Slater-Usoh-Steed Questionnaire as an instrument of self-reported for assessment of sense of presence caused. The overall results of this investigation suggest that 3D Affective Induction Scenarios induces an increased sense of presence, compared to their counterparts presented in 2D technology. We also verified that scenario unpleasant valence provokes greater sensation of presence compared to pleasant and neutral scenarios.

Keywords: presence; virtual reality; 3D affective induction scenarios; condition emotional.

\section{RESUMEN}

La Realidad Virtual (RV) ha venido a ser utilizada en la inducción de emociones, y ha sido asociada a respuesta emociónales de mayor validez ecológica. La presencia, mientras experiencia de "estar allá", en un determinado ambiente mediado, está íntimamente asociada a la RV. El presente trabajo tiene el objetivo de verificar, si los Escenarios de Inducción Afectiva-3D inducen una mayor sensación de presencia comparativamente a sus homólogos presentados con la tecnología 2D. Presentamos a 30 participantes sanos los Escenarios de Inducción Afectiva en ambas modalidades de visualización (2D vs. 3D), y simultáneamente se registraron mediciones psicofisiológicas de la Actividad Electrodérmica y de la Taxa Cardíaca y, a final de cada escenario, se utilizó la escala de dominancia del Self-Assessment Manikin adaptada para medir la presencia, y él Slater-Usoh-Steed Questionnaire, como instrumento de auto-relato para evaluación de la sensación de presencia provocada. Los resultados globales sugieren Escenarios de Inducción Afectiva - 3D induce una mayor sensación de presencia, comparativamente a sus homólogos presentados en la tecnología 2D. Verificamos aún que el escenario de valencia desagradable provoca mayor sensación de presencia comparativamente con los escenarios de valencia agradable y neutro.

Palabras clave: presencia; realidad virtual; escenarios de inducción afectiva -3D; condición emocional. 


\section{Introduçáo}

Nos últimos anos, a tecnologia da Realidade Virtual (RV) tem vindo a ser aplicada em diversos domínios da Psicologia, nomeadamente no tratamento no confronto de pacientes com situaçóes fóbicas (Lopéz \& Lizandra, 2009; Costa, 2006) perturbação de pânico (Carvalho, Freire, \& Nardi, 2008), perturbaçóes de ansiedade (Anderson \& Price, 2007), no tratamento de perturbaçóes alimentares (Botella et al., 2007; Riva, Bacchetta, Baruffi, Rinaldi, \& Molinari, 1999), nas perturbaçóes espectro autista (Strickland, 1997), na recuperação cognitiva de doentes com traumatismo crânio-encefálico (Christiansen et al., 1998) e como potencial analgésico em pacientes sujeitos a procedimentos dolorosos (Hoffman, Patterson, Carrougher, \& Sharar, 2001).

A RV tem sido descrita como sendo um conjunto de tecnologias computorizadas que, quando combinadas, estabelecem uma relaçáo com o mundo gerado por computador de tal forma convincente que o utilizador considera que está realmente num ambiente tridimensional (Seabra \& Santos, 2005).

$\mathrm{Na}$ verdade, já Riva et al. (2007) referiam que os ambientes virtuais estabelecem um meio de comunicação afetiva e eficaz na indução de respostas emocionais, comportamentais e fisiológicas coerentes com o conteúdo do ambiente recriado, constituindo-se, desse modo, como um bom método para avaliar as alteraçōes dinâmicas dessas mesmas respostas em contexto experimental.

Nos anos mais recentes, as tecnologias de RV têm apresentado progressos inovadores e particularmente a tecnologia $3 \mathrm{D}$ tem-se tornado cada vez mais acessível, oferecendo-nos a possibilidade de visualizar estímulos de uma forma mais aproximada daquela que experimentamos no nosso dia-a-dia. Assim, a utilização da tecnologia RV permite o desenvolvimento de estímulos com propriedades mais próximas da realidade, na medida em que a tridimensionalidade torna-se fundamental para conferir maior sensação de presença, possibilitando uma melhor emulação da realidade em ambiente laboratorial (Insko, 2003).

A literatura especializada tem definido a presença virtual como um estado de consciência que permite ao indivíduo sentir a sensaçáo subjetiva (Slater, 2003) de estar fisicamente presente no local projetado mais do que no local onde se encontra efetivamente (Freeman, Avons, Pearson, \& IJsselsteijn, 1999). Para Sanchez-Vives e Slater (2005), o termo presença é considerado como a propensão das pessoas para responderem a estímulos de forma proporcional à forma como seriam capazes de se comportar se esses mesmos estímulos fossem reais. $\mathrm{Na}$ mesma linha, Zahorik e Jenison (1998) sugeriram que existe presença quando as açôes do participante no ambiente são consistentes com as respostas genuínas, 
isto é, as respostas que teria no mundo real. Tendo em consideração que a grande maioria da investigação neste domínio considera a presença como sendo um estado subjetivo (Slater, 2003), tem sido frequentemente avaliada por meio de questionários de autorrelato (Slater, 2002; Slater, 2009; Slater, Lotto, Arnold, \& Sanchez-Vivez, 2009), que dependem essencialmente da memória decorrente da experiência. Por conseguinte, de modo a ser feita uma avaliação contínua da experiência, introduziram-se medidas mais objetivas, nomeadamente a utilização de medidas fisiológicas periféricas (Insko, 2003). Nesta ordem de ideias, alguns investigadores (Meehan, 2001; Riva, Wiederhold, \& Molinari, 1998; Wiederhold, Gevirtz, \& Spira, 2001), destacam a medição do Ritmo Cardíaco (RC) e a medição das alterações da Actividade Electrodérmica (AED) como os sinais mais utilizados. Na verdade, Meehan (2001) comprovou a existência de uma relação directamente proporcional entre desaceleração cardíaca e elevados níveis de presença. Por esse motivo, o RC constitui-se como sendo uma medida fisiológica periférica empiricamente validada para o estudo da sensação de presença (Meehan, 2001). O mesmo autor considera que a AED também apresenta potencial para o estudo da sensação de presença, mas não se revela tão discriminativa como o RC.

No seguimento do estudo de Monteiro, Barbosa e Silvério (2011), que defendem a pertinência de ser realizada uma investigação direcionada para o estudo da sensação de presença, o presente trabalho tem como objetivo verificar, por meio de medidas subjectivas, nomeadamente questionários após experiência e por meio de medidas fisiológicas periféricas, em particular através da AED e RC, a hipótese segundo a qual a visualização dos Cenários de Indução Afetiva 3D induz uma maior sensação de presença pelos mesmos cenários apresentados com a tradicional tecnologia 2D. De forma adicional pretendíamos ainda saber se adaptação da escala dominância do Self-Assessment Manikin-SAM é adequada para a avaliação da presença.

\section{Método}

\section{Amostra}

Recrutamos 30 participantes saudáveis, todos do sexo masculino, por uma questão de simplificar variáveis, uma vez que se assim não fosse teríamos que estar atentos a outras variáveis nomeadamente hormonais (Andreassi, 2006), com idades compreendidas entre os 18 e os 50 anos $(M=22.10$; $D P=6.88)$. Foram considerados critérios de exclusão a presença de diagnósticos psiquiátricos ou consumo 
de substâncias de abuso, de acordo com o DSM-IV-TR (American Psychological Association, 2002), e, ainda, a presença de deficiências visuais não compensadas que pudessem comprometer o desempenho nas tarefas experimentais.

\section{Materiais}

Medidas de apreciação subjetiva

A forma mais simples de avaliar a experiência subjetiva consiste em perguntar diretamente ao indivíduo em que medida determinado estímulo ou evento é agradável ou desagradável e qual a intensidade do estado emocional sentido. Trata-se, portanto, de uma técnica baseada no autorrelato, mas que pode ser aperfeiçoada através da aplicação dos métodos psicométricos de construção de escalas. Por exemplo, o Self Assessment Manikin - SAM , desenvolvido por Lang (1980) com cuidados psicométricos, é especialmente adequado para estudar a vertente experiencial da resposta emocional, uma vez que é livre de influências culturais e não dispensa a conduta verbal (Bradley \& Lang, 1994). Requer apenas papel e lápis e permite a avaliação das três dimensóes emocionais - valência, intensidade e controlo - através de três escalas pictográficas com figuras humanoides. Além de proporcionar informaçáo relativa a cada uma das dimensóes de forma não verbal, a sua aplicação é simples e rápida (Lang, Bradley, \& Cuthbert, 2008). De forma a medir os autorrelatos da presença, adaptou-se a escala pictográfica do SAM (Lang, 1980), particularmente a escala dominância para proceder à mediçâo da sensação de presença. $\mathrm{Na}$ adaptação para a sensação de presença, cada resposta é avaliada numa escala graduada de 9 níveis variando entre o extremo mínimo ( 1 = pouco presente), até ao extremo máximo $(9=$ muito presente). Importa referir que a presença é geralmente considerada como uma variável contínua, de modo que pode-se atingir diferentes graus de presença numa experiência (Slater et al., 2009). Nesta linha de raciocínio, IJsselsteijn, Ridder, Hamberg, Bouwhuis e Freeman (1998) constataram que o nível de presença relatado varia consideravelmente ao longo do tempo, dependendo do conteúdo emocional exibido no material estimulante.

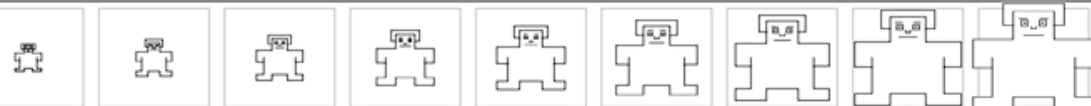

Figura 1. Escala Presença - SAM 
Foi também utilizado o Slater-Usoh-Steed Questionnaire - SUS formulado por Slater, Usoh e Stedd (1994). Esse questionário compreende seis questóes, em que cada uma das respostas abrange uma escala de 1 a 7 , variando entre o extremo mínimo ( 1 = baixa presença), até ao extremo máximo ( 7 = elevada presença). Cada uma das seis questóes envolve aspectos indicadores da presença, tais como a sensação de estar no cenário apresentado, de este se tornar uma realidade e de ser recordado como um lugar visitado. Desta forma, cada uma das seis questóes envolve uma escala de 1 a 7 , em que pontuaçóes mais altas se reportam para níveis de presença superiores. Na literatura especializada, vários estudos foram realizados no âmbito da avaliação da sensação de presença com recurso ao questionário SUS, aludindo para a sensibilidade deste método de medição, de forma que é apontada como uma medida capaz de distinguir vários níveis de presença (Khanna, Yu, Mortesen, \& Slater, 2006; Slater, Usoh, \& Steed, 1994; Usoh et al., 1999; Usoh, Catena, Arman, \& Slater, 2000; Youngblut \& Huie, 2003).

Quadro 1. Slater - Usoh - Steed Questionnaire (SUS).

1. Até que ponto se sentiu presente no cenário apresentado?

1 a 7 , em que 1 corresponde a uma experiencia normal, e 7 o sentido de estar presente no cenário.

2. Até que ponto cenário apresentado se tornou uma realidade e quase se esqueceu do contexto laboratorial em que decorre a experiência?

1 a 7 , em que 1 corresponde nunca, e 7 sempre.

3. Até que ponto ao recordar-se do cenário, o vê como uma imagem, ou como um sitio onde esteve presente?

1 a 7, em que 1 apenas como imagem, e 7 como um sítio em que esteve presente.

4. Durante o tempo da experiência, foi mais forte o sentido de estar no cenário ou de estar em outro lugar?

1 a 7 , em que 1 corresponde a estar em outro lugar, e 7 estar no cenário.

5. Em que medida as condições estruturais (cor, objetos) do cenário o recordam de outras situações semelhantes em que já esteve?

1 a 7 , em que 1 equivale pouco, e 7 muito.

6. Durante algum momento da experiência pensou que estivesse realmente no cenário?

1 a 7 , em que 1 equivale a pouco, e 7 muito.

1. Até que ponto se sentiu presente no cenário apresentado?

1 a 7 , em que 1 corresponde a uma experiencia normal, e 7 o sentido de estar presente no cenário. 
2. Até que ponto cenário apresentado se tornou uma realidade e quase se esqueceu do contexto laboratorial em que decorre a experiência?

1 a 7 , em que 1 corresponde nunca, e 7 sempre.

3. Até que ponto ao recordar-se do cenário, o vê como uma imagem, ou como um sitio onde esteve presente?

1 a 7, em que 1 apenas como imagem, e 7 como um sítio em que esteve presente.

4. Durante o tempo da experiência, foi mais forte o sentido de estar no cenário ou de estar em outro lugar?

1 a 7 , em que 1 corresponde a estar em outro lugar, e 7 estar no cenário.

5. Em que medida as condições estruturais (cor, objetos) do cenário o recordam de outras situações semelhantes em que já esteve?

1 a 7 , em que 1 equivale pouco, e 7 muito.

6. Durante algum momento da experiência pensou que estivesse realmente no cenário?

1 a 7 , em que 1 equivale a pouco, e 7 muito.

Medidas fisiológicas periféricas

Para estudar a componente fisiológica, é necessário recorrer aos modelos e métodos da psicofisiologia, uma vez que esta disciplina científica permite quantificar as respostas fisiológicas subjacentes a um determinado comportamento. De fato, uma manifestação característica dos fenômenos emocionais é a ocorrência de um conjunto de alteraçóes corporais internas de que são exemplo a sudação, a variação de pressão sanguínea ou alteração do ritmo cardíaco. Pois bem, estas alterações corporais correspondem a um conjunto de modificaçóes fisiológicas cujo registro objetivo só é possível mediante a utilização de técnicas psicofisiológicas (Castellar, 2000).

Desta forma, a aquisição das medidas fisiológicas periféricas, nomeadamente da AED e do RC, foi realizada simultaneamente desde o princípio ao final do protocolo experimental, mas o início de cada ensaio (i.e., de cada cenário, apresentado em 2D ou 3D) foi devidamente assinalado no registo para posterior análise.

Esta aquisição foi assegurada por um polígrafo eletrônico da marca $B I O$ PAC (Goleta, USA), modelo MP100, equipado com um amplificador monocanal para registro da AED, modelo GSR100C (corrente contínua e voltagem constante) e um amplificador de sinal fotopletismográfico, modelo PPG100C, para registro do RC. Utilizaram-se dois eletrodos $A g-A g / C l$ reutilizáveis, não polarizáveis, modelo TSD203 para captação da AED e um transducer fotoelétrico, modelo TSD100, para captação do RC (pulso radial) via alterações da pressão sanguínea. 


\section{Procedimentos}

Neste estudo utilizou-se um desenho experimental 2 Modos de Visualização (2D vs. 3D) x 3 Condiçôes Emocionais (Agradável, Neutra, Desagradável), intrassujeitos. O protocolo experimental incluiu que cada participante observasse três cenários em cada modo de visualização enquanto eram recolhidas as respostas fisiológicas. Após a visualização os participantes avaliavam a sensação de presença relativa ao cenário que acabaram de visualizar.

A recolha de dados foi realizada em sessóes individuais, no Laboratório de Psicofisiologia, do Instituto Superior Ciências Saúde - Norte, Gandra, Portugal. Após entregue o Consentimento Informado, os participantes foram convidados a sentarem-se confortavelmente na cadeira situada de frente para o monitor em que seriam exibidos os cenários. Instruíram-se os participantes a responder ao SAM e ao SUS, e a prestar atenção a cada cenário apresentado. Posteriormente, procedeu-se à colocação do transducer para a fotopletismografia na face palmar da falange distal do dedo indicador da mão nâo dominante e dos eletrodos para a $\mathrm{AED}$ na face palmar da falange distal do dedo médio e indicador da máo dominante.

O polígrafo foi operado a partir de um computador com MAC OS X Lion, onde se encontrava instalado o software para registro e análise Acqknowledge Versão 3.7.1, também da marca BIOPAC. Na montagem dos eletrodos foi aplicado um gel electrolítico hipossaturado e isotônico, com a referência GEL 100.

Foram utilizados três cenários de RV, designados de Cenários de Indução Afetiva 3D, cada um dos quais integrando um conjunto de 15 estímulos, de modo a tornar possível a manipulação de três condições de indução emocional (Agradável, Neutra, Desagradável) (Monteiro et al., 2011). Estes estímulos que constituíram cada um dos cenários foram criteriosamente selecionados a partir do trabalho de normalizaçáo realizado por Monteiro et al. (2011) com base nas avaliaçóes de intensidade e valência reportadas por 214 participantes.

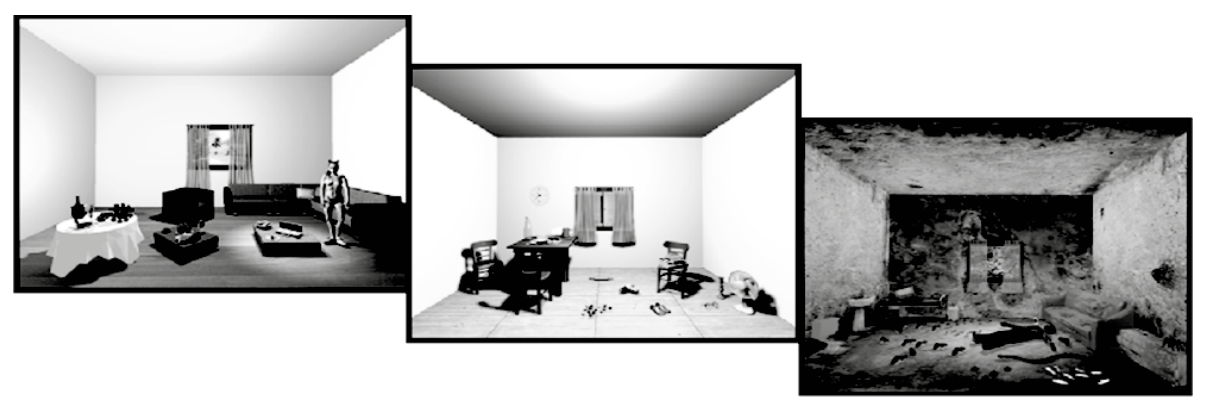

Figura 2. Representação dos cenários CIA-3D

(Monteiro, Barbosa, \& Silvério, 2011) 
Os estímulos visuais apresentados consistiram nos CIA 3D (Monteiro et al., 2011), em duas modalidades de visualização (2D vs. 3D), foram apresentados durante $10 \mathrm{~s}$ e de seguida surgia a instrução para a tarefa de classificação assim como cada uma das escalas de presença do SAM e do SUS. A avaliação de cada estímulo foi reportada de forma verbal em cada uma das escalas. O protocolo de estimulação foi organizado em onze ensaios por participante, correspondentes a cada uma das condiçóes emocionais (Agradável, Neutro, Desagradável) apresentadas em dois formatos de visualização (2D e 3D), com a seguinte organização: um ponto de fixação $(5 \mathrm{~s})$, seguia-se a apresentação de cada cenário $(10 \mathrm{~s})$ em $2 \mathrm{D}$ ou 3D, terminado o tempo de exposição seguia-se a instrução para responder à escala de presença do SAM e imediatamente de seguida a do SUS. Finalizado o ensaio, sucedia-se um novo ponto de fixação $(5 \mathrm{~s})$.

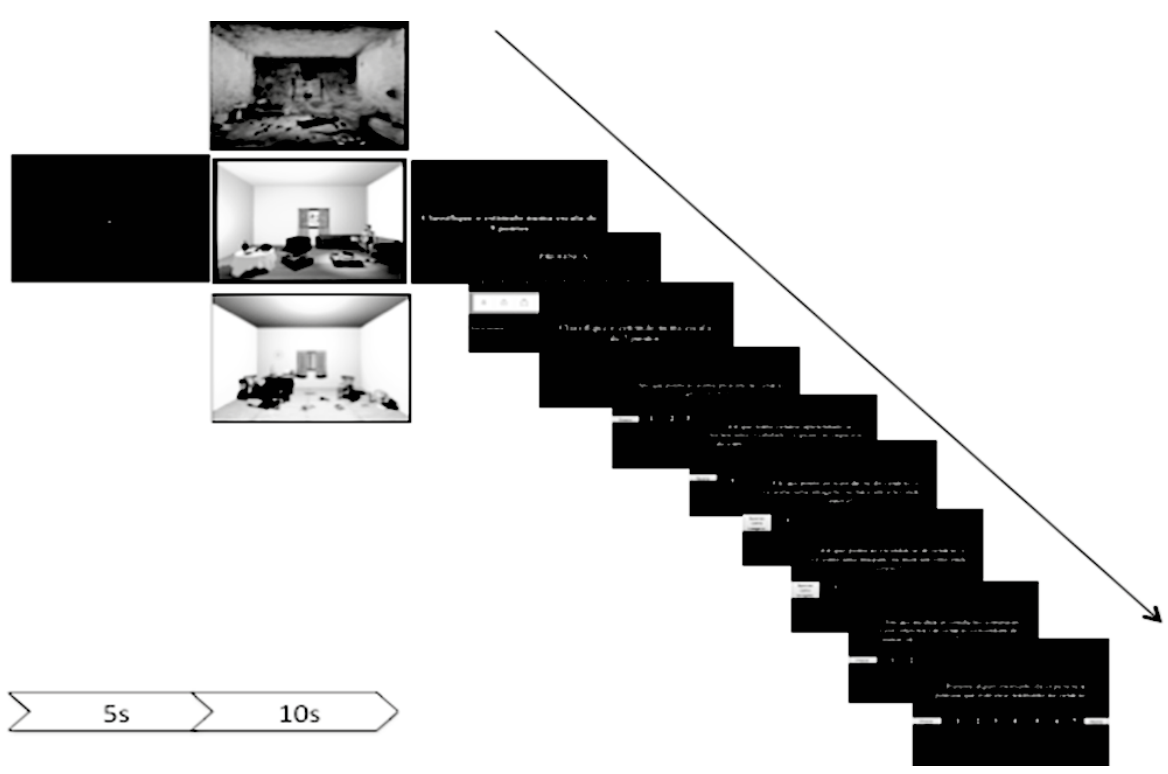

Figura 3. Protocolo de estimulação com os cenários do CIA-3D, visualizados em $2 \mathrm{D}$ e $3 \mathrm{D}$ através projeção estereoscópica passiva

Para administração dos estímulos, foi utilizado o software Presentation 0.71 (Neurobehavioral Systems, Inc.) instalado num computador com o sistema operativo $M A C O S X$, com ecrã de 15 polegadas. Para a visualizaçáo dos cenários em $3 \mathrm{D}$ recorremos ao sistema de $\mathrm{RV}$ com visualização estereoscópica passiva, nomeadamente ao método anaglifo. O sistema anaglifo é extremamente fácil de recriar 
e não necessita de instrumentos especiais de projeção ou visualização (Boulos $\&$ Robinson, 2009; Seabra \& Santos, 2005; Zelle \& Figura, 2004).

A sequência dos ensaios foi aleatorizada entre os participantes de modo a controlar o efeito de ordem. A resposta às escalas é feita oralmente, no sentido de prevenir movimentos que pudessem provocar artefatos motores no registro do polígrafo. Simultaneamente foi realizada a aquisição das medidas fisiológicas periféricas, nomeadamente da AED e do RC, desde o princípio ao fim do protocolo experimental, salientado que no início de cada ensaio tanto $2 \mathrm{D}$ como 3D foi devidamente assinalado no registro para posterior análise.

\section{Resultados}

Para análise de dados recorreu-se ao software SIGMA PLOT - Versão 12.1. (Systat Software, Chicago, USA). Para estudar o efeito da Condição Emocional e do Modo de Visualização sobre as medidas dependentes obtidas, nomeadamente as pontuaçóes das escalas de presença do $S A M$ e $S U S$, recorreu-se a uma análise de variância de medidas repetidas a dois factores (Two Way ANOVA) reportando a um nível de significância de $p \leq .05$. Sempre que necessário, procedeu-se a comparaçóes múltiplas através de análise post-hoc com o Teste de Holm-Sidak.

Considerando a presença SAM, encontramos um efeito principal quer para a Condição Emocional $\left(F_{[2,179]}=4.472, p<.013\right)$, quer para o Modo de Visualização $\left(F_{[1,179]}=89.588, p<.001\right)$ e um efeito da interação entre as duas variáveis referidas $\left(F_{[2,179]}=10.317, p<.011\right)$. A análise post-hoc com o Teste Holm-Sidak revelou níveis de presença estatisticamente superiores no cenário desagradável 3D quando comparados com os seus equivalentes no modo de visualização $2 \mathrm{D}\left(t_{(179)}\right.$ $=7.807, p<.001)$. O mesmo se verificou no cenário agradável $3 \mathrm{D}$ em comparação com o cenário agradável no modo de visualização $2 \mathrm{D}\left(t_{(179)}=5.031, p<.001\right)$. Foram ainda encontradas diferenças significativas entre os cenários neutros $3 \mathrm{D}$ e $2 \mathrm{D}\left(t_{(179)}=3.556, p<.001\right)$.

Abordando a presença para a primeira questão do SUS, encontramos um efeito principal para a Condição Emocional $\left(F_{[2,179]}=7.648, p<.001\right)$ e para a interação entre esta variável e o Modo de Visualização $\left(F_{[2,179]}=5.560, p<.005\right)$, tendo-se também encontrado um efeito significativo para este último $\left(F_{[1,179]}=\right.$ $=130.825, p<.001)$. A análise post-hoc com o Teste Holm-Sidak revelou níveis de presença estatisticamente superiores para o cenário desagradável no modo de visualização $3 \mathrm{D}$ em comparação com o cenário desagradável no modo de visualização $2 \mathrm{D}(t=8.940, p<.001)$. O cenário agradável projetado em $3 \mathrm{D}$ produziu 


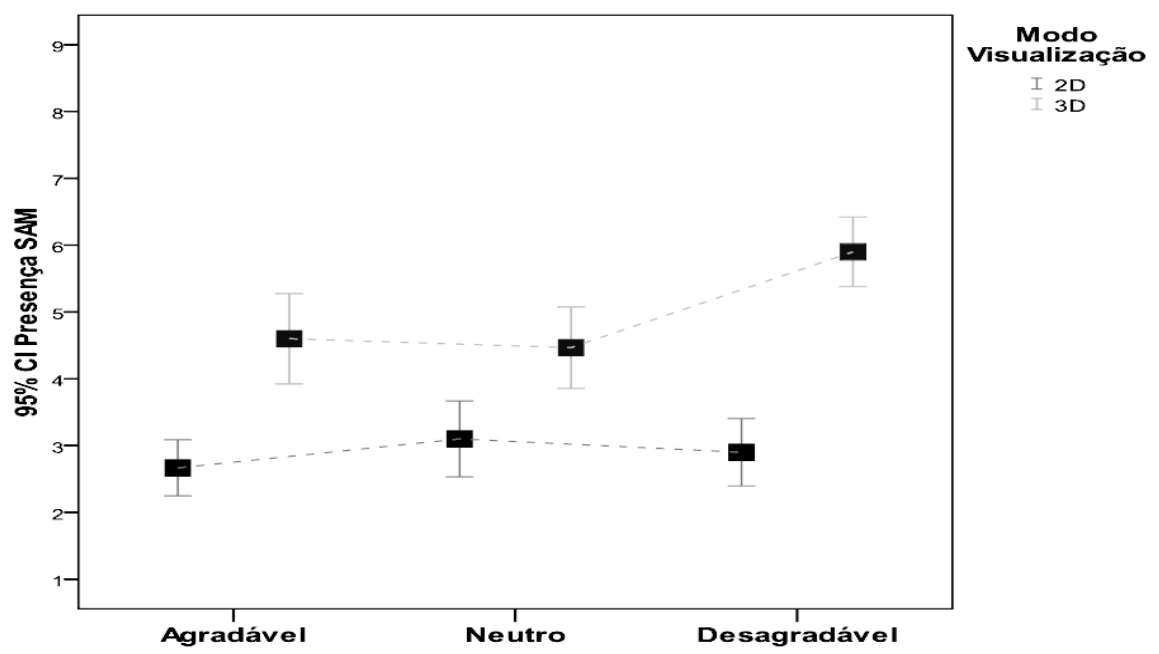

Figura 4. Médias e Desvios Padrão para medição da presença dos cenários de indução afetiva, em função do modo de visualização, 2D vs. 3D para o SAM. Agradável 2D 2.67 (1.12) vs. Agradável 3D 4.60 (1.81); Neutro 2D 3.10 (1.52) vs. Neutro 3D 4.47 (1.63); Desagradável 2D 2.90 (1.35) vs. Desagradável 3D 5.90 (1.40).

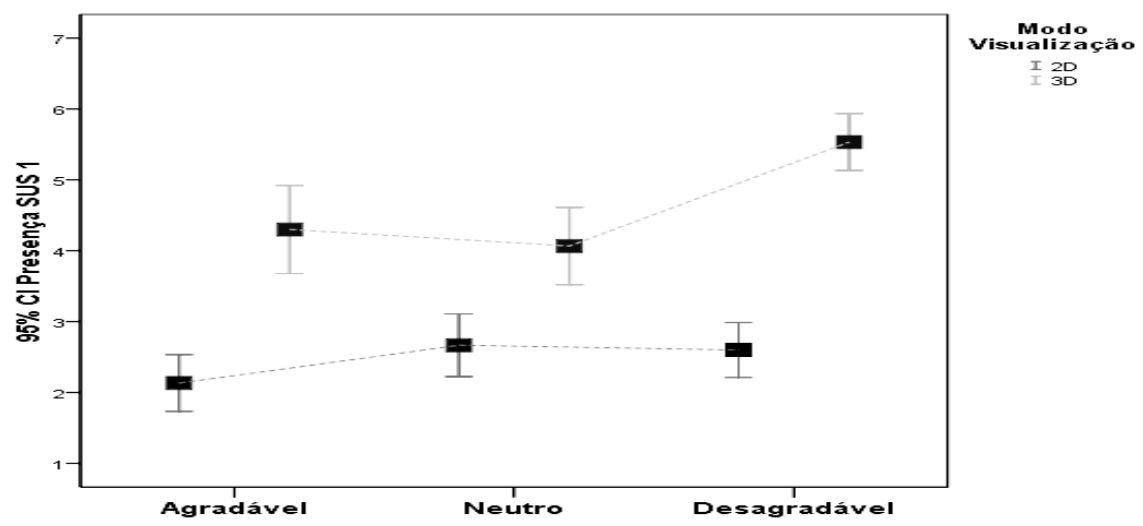

Figura 5. Médias e Desvios Padrão para medição da presença dos cenários de indução afetiva, em função do modo de visualização, 2D vs. 3D para SUS 1. Agradável 2D 2.13 (1.07) vs. Agradável 3D 4.30 (1.66); Neutro 2D 2.67 (1.84) vs. Neutro 3D 4.07 (1.56); Desagradável 2D 2.60 (1.04) vs. Desagradável 3D 5.53 (1.07). 
igualmente níveis mais elevados de presença do que o cenário agradável visualizado em 2D $(t=6.604, p<.001)$. Relativamente ao cenário neutro, foram igualmente verificadas diferenças estatisticamente significativas entre o cenário neutro no modo de visualização 3D e o seu equivalente $2 \mathrm{D}(t=4.267, p<.001)$.

No nível segunda questão do SUS, encontramos um efeito principal para a Condição Emocional $\left(F_{[2,179]}=4.524, p<.012\right)$ e para o Modo de Visualização $\left(F_{[1,179]}=82.713, p<.001\right)$, tendo-se também encontrado um efeito significativo na interação entre essas duas variáveis $\left(F_{[2,179]}=3.232, p<.042\right)$. Através da análise post-hoc com o Teste Holm-Sidak observaram-se níveis de presença estatisticamente superiores para o cenário desagradável no modo de visualização $3 \mathrm{D}$ em comparação com o cenário desagradável no modo de visualização $2 \mathrm{D}(t=7.300$, $p<.001)$. O cenário agradável projetado em 3D produziu igualmente níveis mais elevados de presença do que o cenário agradável visualizado em $2 \mathrm{D}(t=4.514, p<$ .001). Bem como o cenário neutro, sendo averiguadas diferenças estatisticamente significativas entre o cenário neutro no modo de visualização $3 \mathrm{D}$ e o seu equivalente $2 \mathrm{D}(t=3.938, p<.001)$.

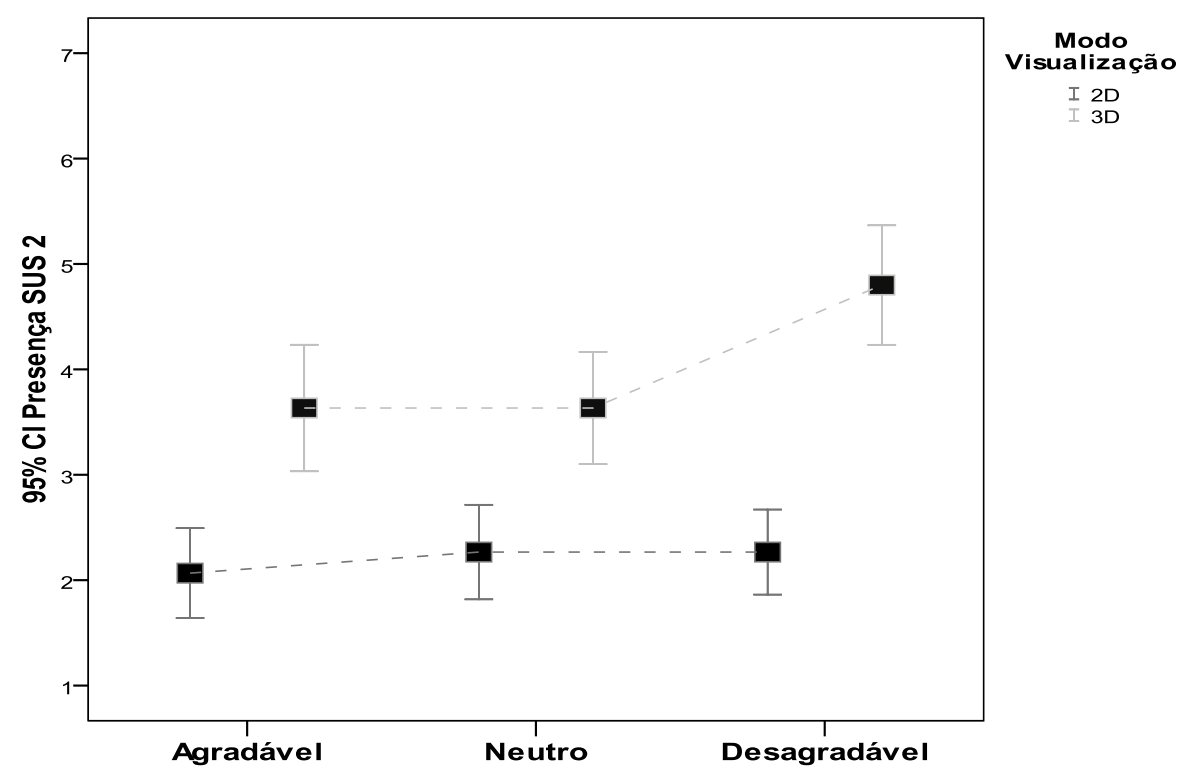

Figura 6. Médias e Desvios Padrão para medição da presença dos cenários de indução afetiva, em função do modo de visualização, $2 \mathrm{D}$ vs. 3D para SUS 2. Agradável 2D 2.07 (1.14) vs. Agradável 3D 3.63 (1.61); Neutro 2D 2.27 (1.20) vs. Neutro 3D 3.63 (1.43); Desagradável 2D 2.27 (1.08) vs. Desagradável 3D 4.80 (1.52). 
Tendo em consideração a presença para a terceira questão do SUS, deparamo-nos com um efeito principal para o Modo de Visualização $\left(F_{[1,179]}=61.335\right.$, $p<.001)$ e para a interaçáo entre esta variável e a Condiçáo Emocional $\left(F_{[2,179]}=\right.$ $6.285, p<.002)$, embora não se tenha encontrado um efeito significativo para este último $\left(F_{[2,179]}=2.439, p<.090\right)$. Pela análise post-hoc com o Teste Holm-Sidak, verificou-se níveis de presença estatisticamente significativos no cenário desagradável 3D $(t=7.101, p<.001)$ e o cenário agradável projetado em 3D produziu igualmente níveis mais elevados de presença do que o cenário agradável visualizado em $2 \mathrm{D}(t=4.370, p<.001)$. Igualmente significativos foram os níveis de presença para o cenário neutro $3 \mathrm{D}$ comparativamente ao seu equivalente em visualização $2 \mathrm{D}(t=2.094, p<.038)$.

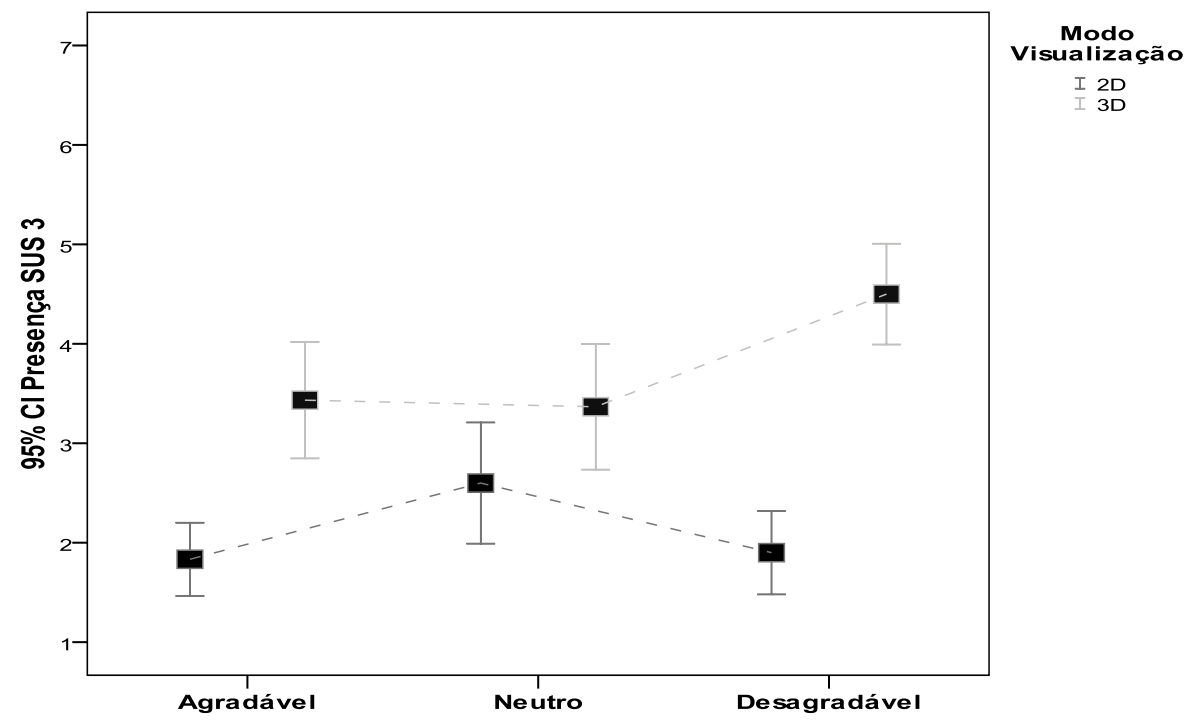

Figura 7. Médias e Desvios Padrão para medição da presença dos cenários de indução afetiva, em função do modo de visualização, 2D vs. 3D para SUS 3. Agradável 2D 2.83 (0.99) vs. Agradável 3D 3.43 (1.57); Neutro 2D 2.60 (1.13) vs. Neutro 3D 3.37 (1.69); Desagradável 2D 1.90 (1.13) vs. Desagradável 3D 4.50 (1.36).

No nível quarta questão do SUS, encontramos um efeito principal para a Condição Emocional $\left(F_{[2,179]}=4.039, p<.019\right)$ e para o Modo de Visualização $\left(F_{[1,179]}==55.481, p<.001\right)$, tendo-se também encontrado um efeito significativo na interação entre estas duas variáveis $\left(\mathrm{F}_{[2,179]}=4.039, p<.019\right)$. Através da análise post-hoc com o Teste Holm-Sidak observou-se níveis de intensidade estatisticamente superiores para o cenário desagradável no modo de visualização $3 \mathrm{D}$ em 
comparação com o cenário desagradável no modo de visualização $2 \mathrm{D}(t=6.539$, $p<.001)$. O cenário agradável projetado em $3 \mathrm{D}$ produziu igualmente níveis mais elevados de intensidade do que o cenário agradável visualizado em $2 \mathrm{D}(t=3.711$, $p<.001)$. Bem como o cenário neutro, sendo averiguadas diferenças estatisticamente significativas entre o cenário neutro no modo de visualização $3 \mathrm{D}$ e o seu equivalente $2 \mathrm{D}(t=2.651, p<.009)$.

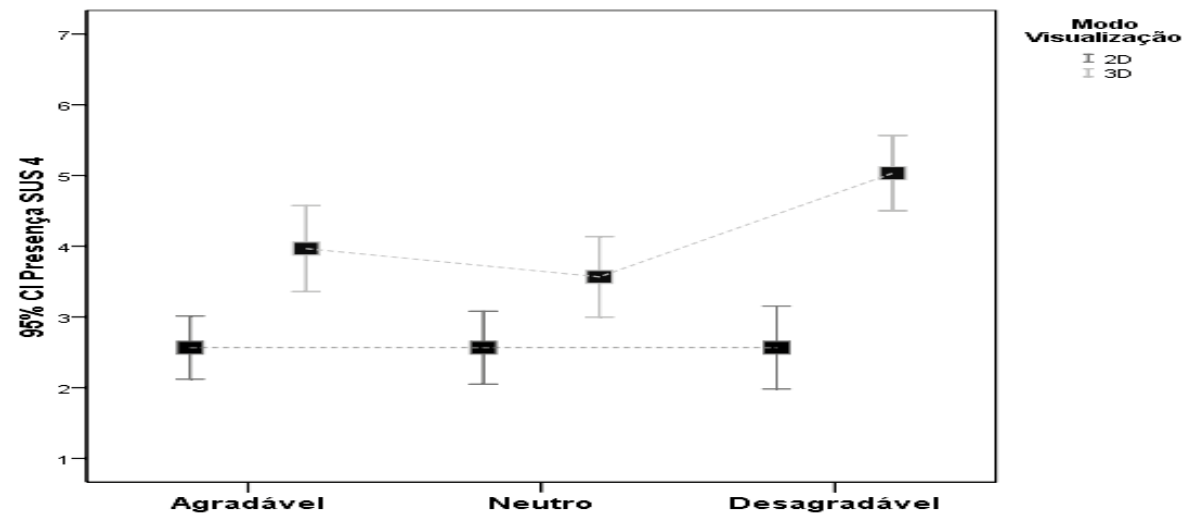

Figura 8. Médias e Desvios Padrão para medição da presença dos cenários de indução afetiva, em função do modo de visualização, 2D vs. 3D para SUS 4. Agradável 2D 2.57 (1.19) vs. Agradável 3D 3.97 (1.63); Neutro 2D 2.57 (1.38) vs. Neutro 3D 3.57 (1.52); Desagradável 2D 2.56 (1.57) vs. Desagradável 3D 5.03 (1.43).

Considerando a presença para a quinta questão do SUS, deparamo-nos com um efeito principal para o Modo de Visualização $\left(F_{[1,179]}=41.160, p<.001\right)$ e para a interação entre esta variável e a Condição Emocional $\left(F_{[2,179]}=4.982, p<\right.$ $.008)$, apesar de não ser deparado um efeito significativo para este último $\left(F_{[2,179]}=\right.$ $1.150, p<.259)$. A análise post-hoc com o Teste Holm-Sidak exibiu níveis de presença estatisticamente superiores para o cenário desagradável no modo de visualização 3D em comparação com o cenário desagradável no modo de visualização 2D $(t=6.277, p<.001)$. Bem como o cenário neutro, sendo averiguadas diferenças estatisticamente significativas entre o cenário neutro no modo de visualização $3 \mathrm{D}$ e o seu equivalente $2 \mathrm{D}(t=2.545, p<.012)$. O cenário agradável projetado em 3D produziu igualmente níveis mais elevados de presença do que o cenário agradável visualizado em $2 \mathrm{D}(t=2.290, p<.023)$. 


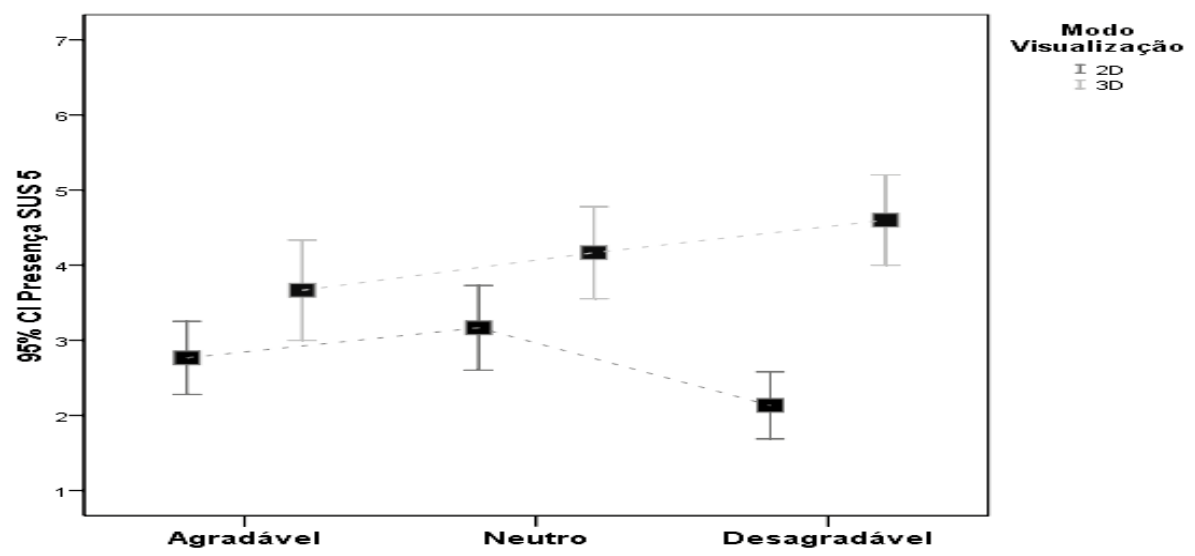

Figura 9. Médias e Desvios Padrão para medição da presença dos cenários de indução afetiva, em função do modo de visualização, 2D vs. 3D para SUS 5. Agradável 2D 2.77 (1.31) vs. Agradável 3D 3.67 (1.79); Neutro 2D 3.17 (1.51) vs. Neutro 3D 4.17 (1.64); Desagradável 2D 2.13 (1.19) vs. Desagradável 3D 4.60 (1.61).

Quanto à sexta questão do SUS, encontramos um efeito principal para o Modo de Visualização $\left(F_{[1,179]}=100.705, p<.001\right)$ embora não se tenha verificado um efeito significativo para a Condição Emocional $\left(F_{[2,179]}=1.217, p<.299\right)$, nem para a interação entre esta variável e o Modo de Visualização $\left(F_{[2,179]}=2.618, p<.076\right)$.

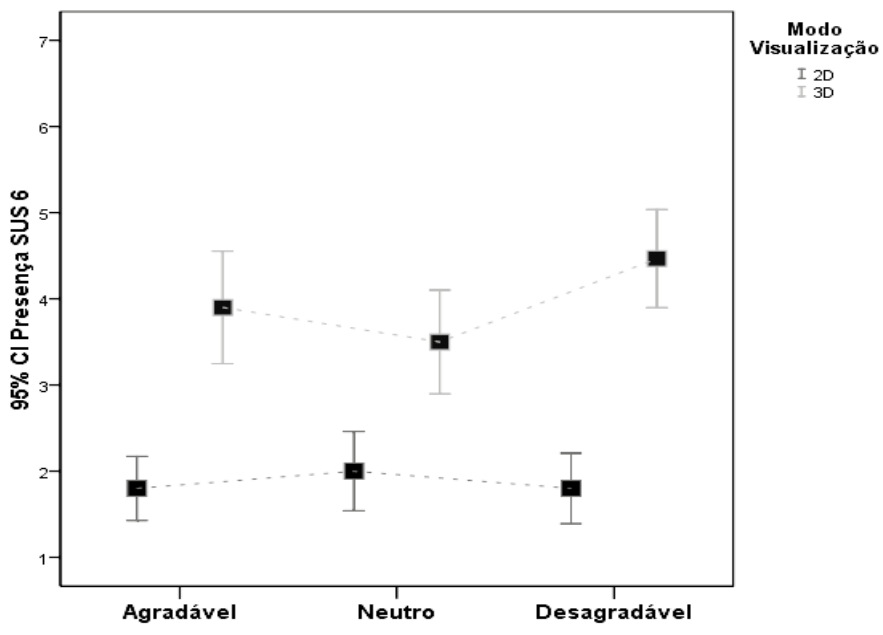

Figura 10. Médias e Desvios Padrão para medição da presença dos cenários de indução afetiva, em função do modo de visualização, 2D vs. 3D para SUS 6. Agradável 2D 1.80 (0.99) vs. Agradável 3D 3.90 (1.75); Neutro 2D 2.00 (1.23) vs. Neutro 3D 3.50 (1.61); Desagradável 2D 1.80 (1.02) vs. Desagradável 3D 4.67 (1.53). 
Passando para a análise dos dados fisiológicos e começando pela AED, consideramos as médias das amplitudes de pico $(\mathrm{em} \mu \mathrm{S})$, visto ser uma das medidas mais discriminativas da ativação simpática (Benedek \& Kaernbach, 2010; Insko, 2003). A ANOVA por medidas repetidas revelou um efeito principal do Modo de Visualização na média das amplitudes de pico da AED $\left(F_{[1,179]}=8.755\right.$, $p<.004)$. Não foram encontradas diferenças para a Condição Emocional $\left(F_{[2,179]}=\right.$ $0.749, p<.0474)$, nem para a interação entre esta variável o Modo de Visualização $\left(F_{[2,179]}=0.0953, p<.909\right)$. O Teste Holm-Sidak revelou uma maior amplitude de pico da AED para o Modo de Visualização 2D $\left(t_{(179)}=2.959, p<.004\right)$, comparativamente com o 3D mas, no que concerne aos cenários Agradável, Neutro e Desagradável, não foram encontradas diferenças significativas.

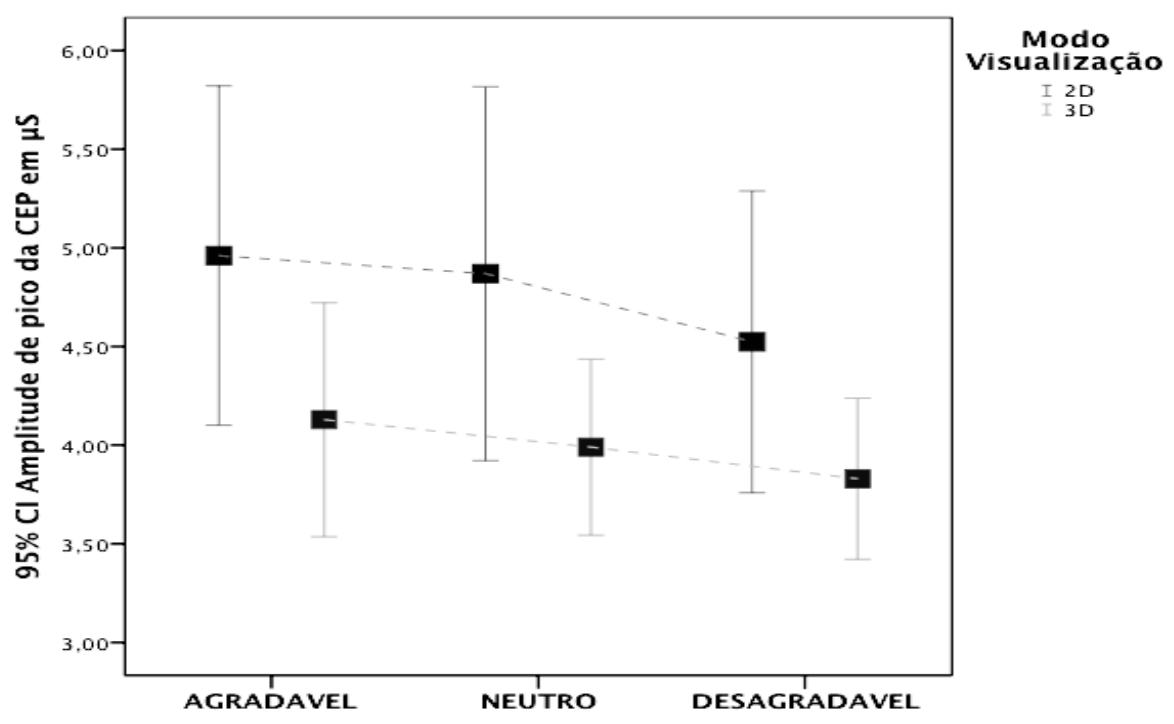

Figura 11. Médias e Desvios Padrão do CEP, resultantes da visualização dos cenários de indução afetiva nas modalidades de visualização $2 \mathrm{D}$ vs. 3D. Agradável 2D 5.11 (2.72) vs. Agradável 3D 4.13 (1.59); Neutro 2D 4.99 (2.85) vs. Neutro 3D 3.99 (1.19); Desagradável 2D 4.53 (2.05) vs. Desagradável 3D 3.82 (1.08).

Por fim, analisamos ainda o RC, medido em batimentos por minuto (BPM) decorrente da visualização dos cenários afetivos nos dois modos de visualização. De acordo com os resultados obtidos, não se revelam efeitos principais do Modo de Visualização nos BPM $\left(F_{[1,179]}=0.0531, p<.818\right)$, nem da Condiçẫo Emocional $\left(F_{[2,179]}=1.047, p<.353\right)$ e também não se encontrou qualquer efeito da interação entre ambos $\left(F_{[2,179]}=0.0125, p<.988\right)$. 
Quadro 2. Médias e Desvios Padrão do RC resultantes da visualizaçáo dos cenários de indução afetiva nas modalidades de visualização $2 \mathrm{D}$ vs. $3 \mathrm{D}$.

\begin{tabular}{cccc}
\hline \multicolumn{4}{c}{ BPM dos CIA 3D } \\
\hline & $\begin{array}{c}\text { Agradável } \\
\text { Média }(\boldsymbol{D P})\end{array}$ & $\begin{array}{c}\text { Neutro } \\
\text { Média }(\boldsymbol{D P})\end{array}$ & $\begin{array}{c}\text { Desagradável } \\
\text { Média }(\boldsymbol{D P})\end{array}$ \\
2D & $80.478(10.80)$ & $80.345(12.57)$ & $77.467(11.54)$ \\
3D & $81.030(14.65)$ & $80.364(12.66)$ & $78.143(10.09)$ \\
\hline
\end{tabular}

\section{Discussão}

Relativamente às medidas de apreciação subjetiva, os resultados revelam que os Cenários de Indução Afetiva 3D provocam maior sensação de presença do que os seus homólogos projetados em 2D, e por isso, estáo de acordo com a literatura especializada, recente (Baños et al., 2004; Freeman \& Avons, 2000; Slater et al., 2009; Usoh et al., 2000; Wideström, Axelsson, Shroeder, Nilsson, \& Aeblin, 2000).

Numa observação mais discriminativa, quando manipulamos a Condição Emocional verificamos que o cenário de valência desagradável provoca maior sensação de presença comparativamente com os cenários de valência agradável e neutro. Estes resultados também estáo de acordo com a literatura, nomeadamente com o estudo de Riva et al., (2007), que averiguou um maior envolvimento emocional e validade ecológica pelos participantes na visualização do cenário de caráter desagradável em comparação ao cenário neutro. Sendo também apurado, através análise post-hoc com o Teste Holm-Sidak, que em todas as questóes, de ambas as medidas de apreciação subjetiva foi encontrado efeito significativo na interação entre a Condição emocional e o Modo de Visualização, suportando os resultados do estudo de Baños et al., (2004), demonstrando que tanto o conteúdo afetivo como o modo de visualização têm um efeito importante sobre o sentido de presença, não sendo esta uma função direta da imersão, dependendo do conteúdo do cenário apresentado (Riva et al., 2007; Schubert, Friedmann, \& Regenbrecht, 2001).

Relativamente aos dados decorrentes das medidas fisiológicas periféricas, pela análise das pontuaçóes médias da amplitude de pico da AED, verificamos um efeito principal para o Modo de Visualização, com a estimulação por tecnologia 2D a provocar amplitudes de pico da AED mais elevadas. Quanto ao RC não foram observadas diferenças estatisticamente significativas, podendo apenas 
notar-se uma tendência para que os cenários apresentados no modo de visualização 3D se associem a um maior RC que os seus equivalentes em $2 \mathrm{D}$ de forma consistente na condição agradável, neutra e desagradável, mas sem que tais diferenças tenham significado estatístico.

De forma ilustrativa, os estudos de Bradley (2000) e Moratti, Keil e Stolarova (2004) demonstraram uma ligeira desaceleração cardíaca associada à indução de estados emocionais que, em concomitância com o aumento da AED, traduz um reflexo de orientação que significa a maior focalização da atenção nos estímulos relacionados com esse padrão de resposta fisiológica. Os investigadores consideram que a presença está relacionada com a alocação de recursos atencionais do participante. Se esses recursos estão concentrados no mundo virtual, o participante sentir-se-á presente nesse mesmo ambiente. Portanto, a monitorização da distribuição de recursos atencionais pode servir para estimar a sensação de presença alcançada pelo sujeito.

Face o exposto, os resultados encontrados parecem não suportar as conclusóes do estudo realizado por Meehan (2001) e Wiederhold et al. (2001), na medida em que seria de esperar que os participantes manifestassem valores mais elevados de AED e, eventualmente, uma desaceleração cardíaca mais pronunciada durante a exposição aos Cenários de Indução Afectiva 3D.

Há, contudo, um aspeto a exigir maior cuidado interpretativo desses resultados. Na literatura sobre a avaliação da sensação de presença através da utilização de medidas fisiológicas periféricas, tem sido majoritariamente utilizado um sistema de visualização Head Mounted Display (HMD) ou CAVE e Teatro Virtual. Pelo que, tendo em consideração que na nossa investigação foi utilizado um sistema de RV estereoscópica, nomeadamente o método anaglifo que, apesar de ser um sistema fácil de recrear não necessitando de instrumentos especiais de projeção ou visualização, é considerado um método em que a imagem perde parte das suas características cromáticas originais (Zelle \& Figura, 2004). Desta forma, podemos sugerir que esta divergência dos resultados observados pela análise das medidas fisiológicas periféricas e das investigaçóes já realizadas no âmbito da avaliação da sensação de presença recorrendo a tais medidas poderá ser suportada pelo recurso a diferentes técnicas de projeção. Além disso, alguns autores têm encontrado que as propriedades formais dos estímulos visuais podem influenciar o processamento cognitivo e a resposta emocional evocada por eles (Detenber $\&$ Reeves, 1996; Simons, Detenber, Roedema, \& Reiss, 1999).

Não obstante algumas limitaçóes, podemos inferir que os resultados são bastante satisfatórios e, tomados no seu conjunto, sugerem que Cenários de Indução Afectiva 3D provocam maior sensação de presença comparativamente com 
os seus homólogos apresentados na tecnologia tradicional 2D, confirmando a hipótese que encaminhou este estudo.

Tanto quanto é do nosso conhecimento, este foi o primeiro estudo em que se procurou analisar em que medida os Cenários de Indução Afetiva 3D provocam maior sensaçáo de presença. Sugerimos, assim, que as investigaçóes futuras se focalizem no desenvolvimento de soluçóes tecnológicas que incrementem o realismo dos cenários como a utilização de estímulos 3D reais. Bem como a utilização de estereoscopia de maior qualidade, nomeadamente a ativa.

\section{Referências}

Anderson, P., \& Price, M. (2007). The role of presence in virtual reality exposure therapy. Journal of Anxiety Disorders, 21, 742-751.

Andreassi, J. L. (2006). Psychophysiology: Human behavior and physiological response. Mahwah, NJ: Lawrence Erlbaum Associates.

American Psychological Association (2002). Manual de diagnóstico e estatística das perturbaçōes mentais (DSM-IV-TR). Lisboa: Climepsi.

Baños, R. M., Botella, C., Alcaniz, M., Liano, V., Guerrero, B., \& Rey, B. (2004). Immersion and emotion: Their impact on the sense of presence. CyberPsychology \& Behavior, 7(6), 734-741.

Benedek, M., \& Kaernbach, C. (2010). A continuous measure of phasic electrodermal activity. Jounal of Neuroscience - Methods, 190, 80-91.

Botella, C., Baños, R. M., García-Palacios, A., Quero, S., Guillén, V., \& Marco, H. J. (2007). Monográfico Intervención en salud en la Red. La utilización de las nuevas tecnologías de la información y la comunicación en psicología clínica. UOC Papers: Revista sobre la Sociedad del Conocimiento, 4, 32-41.

Boulos, M. N., \& Robinson, L. R. (2009). Web GIS in practice VII: stereoscopic 3-D solutions for online maps and virtual globes. International Journal of Health Geographic's, 59(8). doi: 10.1186/1476-072X-8-59

Bradley, M. M. (2000). Emotion and motivation. In J. T. Cacioppo, L. G. Tassinary, \& G. G. Berntson (Eds.), Handbook of psychophysiology (pp. 602-642). New York: Cambridge University Press.

Bradley, M. M., \& Lang, P. J. (1994). Measuring emotion: The self-assessment manikin and the semantic differential. Journal of Behavior Therapy and Experimental Psychiatry, 25, 49-59.

Castellar, J. V. (2000). Una introducción a la psicofisiología clínica. Madrid: Ediciones Pirámide.

Carvalho, M. R., Freire, R. C., \& Nardi, A. E. (2008). Realidade virtual no tratamento do transtorno de pânico. Jornal Brasileiro Psiquiatria, 57, 64-69. 
Christiansen, C., Abreu, B., Ottenbacher, K., Huffman, K., Masel, B., \& Culpepper, R. (1998). Task performance in virtual environments used for cognitive rehabilitation after traumatic brain injury. Archives of Physical Medicine and Rehabilitation, 79(8), 888-892.

Costa, R. M. E. M. (2006). A realidade virtual nas neurociências. In R. Tori, C. Kirner, \& R. Siscoutto (Eds.), Fundamentos e tecnologia de realidade virtual e aumentada - Livro do Pré-Simpósio VIII Symposium on Virtual Reality (pp. 327-333), Belém, PA, Brasil, 02 de maio de 2006.

Detenber, B. H., \& Reeves, B. (1996). A bio-informational theory of emotion: Motion and image size effects on viewers. Journal of Communication, 46(3), 66-84.

Freeman, J., \& Avons, S. E. (2000). Focus groups exploration of presence through advanced broadcast services. In E. Rogowitz \& T. N. Pappas (Eds.), Proceedings of the SPIE v3959-Human Vision and Electronic Imaging V (pp. 530-539), San Jose, CA: SPIE. doi: 10.1117/12.387207

Freeman, J., Avons, S. E., Pearson, D. E., \& IJsselsteijn, W. A. (1999). Effects of sensory information and prior experience on direct subjective ratings of presence. Presence: Teleoperators and Virtual Environments, 8, 1-13.

Hoffman, H. G., Patterson, D. R., Carrougher, G. J. \& Sharar, S. R. (2001). The effectiveness of virtual reality based pain control with multiple treatments. Clinical Journal of Pain, 17(3), 229-235.

IJsselsteijn, W. A., Ridder, H., Hamberg, R., Bouwhuis, D., \& Freeman, J. (1998). Perceived depth and the feeling of presence in 3D TV. Displays, 18, 207-214.

Insko, B. E. (2003). Measuring Presence: Subjective, Behavioral and Psychological Methods. In G. Riva, F. Davide, \& W. A. IJsselsteinjin (Eds.), Being there: Concepts, effects and measurement of user presence in synthetic environment (pp. 137-145). Amsterdam: IOS Press.

Khanna, P., Yu, I, Mortesen, J., \& Slater, M. (2006). Presence in response to dynamic visual realism: a preliminary report of an experiment study. ACM Symposium on Virtual Reality Software and Technology (pp. 364-367). Cyprus: ACM VRST 2006.

Lang, P. J. (1980). Behavioral treatment and bio-informational assessment: Computer applications. In J. B. Sidowski, J. H. Johnson, \& T. A. Williams (Eds.), Technology in mental health care delivery systems (p. 119-137). Norwood: Ablex.

Lang, P. J., Bradley, M. M., \& Cuthbert, B. N. (2008). The international affective picture system: Affective ratings of pictures and instruction manual. Technical Report A-8. Gainesville, FL: University of Florida.

Lopéz, D. C. P., \& Lizandra, M. C. J. (2009). Desarrollo de sistemas de realidad virtual y aumentada para la visualización de entornos acrofóbicos. Estudios comparativos entre ellos. Valência, Espańa: Universidad Politecnica de Valência.

Meehan, M. (2001). Physiological reaction as an objective measure of presence in virtual environments. (A dissertation submitted to the faculty of the University of North Carolina at 
Chapel Hill in partial fulfillment of the requirements for the degree of Doctor of Philosophy in the Department of Computer Science. Chapel Hill, North Carolina, United States)

Monteiro, L., Barbosa, F., \& Silvério, J. (2011). Dados normativos da Indução de Emoções em Ambientes de Realidade Virtual. Saúde Mental, 12(6), 10-20.

Moratti, S., Keil, A., \& Stolarova, M. (2004). Motivated attention in emotional picture processing is reflected by activity modulation in cortical attention networks. Neuroimage, 21(3), 954-964.

Riva, G., Bacchetta, M., Baruffi, M., Rinaldi, S., \& Molinari, E. (1999). Journal of Behavior Therapy and Experimental Psychiatry, 30, 221-230.

Riva, G., Mantovani, F., Capideville, C. S., Preziosa, A., Morganti, F., Villani, D., ... Alcañiz, M. (2007). Affective interactions using virtual reality: The link between presence and emotions. CyberPsychology \& Behavior, 10(1), 45-56.

Riva, G., Wiederhold, B. K., \& Molinari, E. (1998). Virtual environments in clinical psychology and neuroscience: Methods and techniques in advanced patient therapist interaction. Amsterdam: IOS Press.

Sanchez-Vives, M. V., \& Slater, M. (2005). From presence to consciousness through virtual reality. Nature Reviews - Neuroscience, 6, 331-339.

Schubert, T., Friedmann, F., \& Regenbrecht, H. (2001). The experience of presence: Factor analytic insights. Presence, 10(3), 266-281.

Seabra, R. D., \& Santos, E. T. (2005). Utilização de técnicas de realidade virtual no projecto de uma ferramenta 3D para o desenvolvimento da habilidade de visualizaçáo espacial. Revista Educação Gráfica, 9, 111-122.

Simons, R. F., Detenber, B. H., Roedema, T. M., \& Reiss, J. E. (1999). Emotion processing in three systems: The medium and the message. Cambridge Journals Online-Psychophysiology, 36, 619-627.

Slater, M. (2002). Presence and the Sixth Sence. Presence, 11(4), 435-439.

Slater, M. (2003). A note on presence terminology. Presence Connect, 3(3), 1-5.

Slater, M. (2009). Place illusion and plausibility can lead to realistic behavior in immersive virtual environments. Philosophical Transactions of the Royal Society of London, 364, 3549-3557.

Slater, M., Lotto, B., Arnold, M. M., \& Sanchez-Vives, M. (2009). How the experience immersive virtual environments: the concept of presence and its measurement. Anuário de Psicologia, 40(2), 193-210.

Slater, M., Usoh, M., \& Steed, A. (1994). Depth of presence in virtual environments. Presence: Teleoperators and Virtual Environments, 3, 130-144.

Strickland, D. (1997). Virtual reality for the treatment of autism. In G. Riva (Ed.), Virtual reality in neuropsychophysiology (pp. 3-12). Amsterdam: IOS Press. 
Wideström, J., Axelsson, A-S., Shroeder, R., Nilsson, A., \& Aeblin, Å. (2000). The collaborative cube puzzle: A comparison of virtual and real environments. In Proceedings of the third international conference on collaborative virtual environments, San Francisco, CA. Pages 165171. doi: $10.1145 / 351006.351035$

Wiederhold, B., Gevirtz, R., \& Spira, J. (2001). Virtual reality exposure therapy vs. imagery desensitization therapy in the treatment of flying phobia. In G. Riva, \& C. Galimberti (Eds.), Towards cyberpsychology: Mind, cognition, and society in the internet age (pp. 254272). Amsterdam: IOS Press.

Usoh, M., Arthur, K., Whitton, M. C., Bastos, R., Steed, A., Slater, M., \& Brooks, F. P. (1999). Walking > Walking-in-Place > Flying, in Virtual Environments. In ACM conference on computer graphics and interactive techniques - SIGGRAPH (pp. 359-364). doi: $10.1145 / 311535.311589$

Usoh, M., Catena, E., Arman, S., \& Slater, M. (2000). Using Presence Questionnaires in Reality. Presence, 9(5), 497-503.

Youngblut, C., \& Huie, O. (2003). The relationship between presence and performance in virtual environments: Results of a VERTS study. Proceedings - IEEE Virtual Reality 2003, 277-278. doi: 10.1109/VR.2003.1191158

Zahorik, P., \& Jenison, R. L. (1998). Presence as being in the world. Presence, 7(1), 78-89.

Zelle, J. M., \& Figura, C. (2004). Simple, low-cost stereographics: VR for everyone. SIGCSE 2004 Proceedings of the 35th SIGCSE technical symposium on Computer science education, 348-352. doi: 10.1145/971300.971421

Recebido em 05 de abril de 2013 Aceito para publicaçấo em 11 de março de 2014

DOI: $10.1590 / 0103-56652015000100008$ 